\title{
Crenças de pacientes diabéticos acerca da terapia nutricional e sua influência na adesão ao tratamento
}

\author{
Beliefs of diabetic patients about nutritional therapy \\ and its influence on their compliance with treatment
}

Flavia M elo Pontieri ${ }^{1}$

M aria M árcia Bachion ${ }^{2}$
${ }^{1}$ Faculdade Latino Americana. Av. Universitária 683, Centro. 75080-150 Anápolis GO . flaviamelo76@hotmail.com ${ }^{2}$ Faculdade deEnfermagem, UniversidadeFederal de Goiás.
Abstract The system of health beliefs influences the process of compliance with diabetes treatment. Compliance with nutritional therapy is one of the greatest challenges found. The purpose of this qualitative approach study was to analyze the beli efs of type 2 diabetic patients regarding nutritional therapy and its influence on the compliance with it. The participants of the study were nine patients being treated in a reference unit for diabetic patients' treatment. The procedures involved were blood exam to check the glycated he moglobin, a questionnaire, semi-structured re corded interview and patient report checking. The analysis of the speeches was made using the thematic categories of Bardin (2000), and the models of Rokeach (1981) and Rosenstock (1974). All participants showed glycated hemoglobin levels higher than $7 \%$, what shows that is difficult for them to control their glicemic levels and possible non compliance. After the analysis of the speeches, two thematic categories were identified: "Recommendations received about feeding" and "Nutritional therapy compliance". Both categories showed authority beliefs and consensus zero, and barriers to nutritional therapy, which the participants see as a restrictive imposition. Those beli efs havenegative influence on the compliance, and so, they have to be considered when providing health assistance to the population.

Key words Diabetes, Patient acceptance of health care, Nutritional therapy
Resumo 0 sistema de crenças em saúde influencia o processo deadesão ao tratamento de diabetes. A adesão à terapia nutricional é um dos maiores desafios. 0 objetivo desseestudo deabordagem qualitativa foi analisar as crenças de pacientes diabé ticos tipo 2 a respeito da terapia nutricional e sua influência na adesão. Participaram do estudo nove pacientes em tratamento em um centro de referência para atendimento à diabéticos. 0 s procedimentos envolvidos foram coleta de sangue para dosagem dehemoglobina glicada, aplicação dequestionário, entrevista semi-estruturada gravada e consulta ao prontuário. A análisedasfalasfoi feita a partir do model o de categorias temáticas de Bardin, esegundo os model os de crenças de Rosenstock e Rokeach. Todos os participantes apresentaram níveis dehemoglobina glicada acima de $7 \%$, denotando dificuldade para o controle glicêmico e possível falta de adesão. Pela análise das falas, foram identificadas duas categorias temáticas: "Recomendações recebidas sobre alimentação" e "Ade são à terapia nutricional". Elas revelam crenças de autoridade e de consenso zero, e de barreiras à terapia nutricional, percebida como imposição restritiva. São crenças que influenciam negativamente a adesão; assim, precisam ser consideradas no atendimento à população.

Palavras-chave Diabetes, Aceitação do paciente de cuidados de saúde, Terapia nutricional 
Introdução

O diabetes mellitus (DM ) podeser definido como uma síndrome de etiologia múltipla, decorrente da falta de insulina e/ou incapacidade da insulina de exercer adequadamente seus efeitos. Caracteriza-se por hipergl icemia crônica com distúrbios do metabolismo de lipídios e proteínas ${ }^{1-3}$. Entre os seus vários tipos, destaca-se o DM tipo 2, que representa $90 \%$ dos casos da síndrome, aparecendo geralmente na idade adulta ${ }^{4}$.

As complicações do DM podem ser agudas ou crônicas. Entre as complicações agudas estão a hiperglicemia ea hipoglicemia. As complicações crônicas podem ser macrovasculares (doença cardíaca coronária, doença vascular periférica e doença cerebrovascular), microvasculares (retinopatia e nefropatia) e neurológicas (neuropatia) ${ }^{5}$.

Atualmente, estima-se que existem em todo o mundo cerca de 150 milhões de pessoas portadoras de diabetes e esse número poderá duplicar até 0 ano de 2025. O DM , juntamente com outras doenças crônicas, atingiu proporções que atualmente permitem considerá-lo como uma pandemia, atingindo todos os países, independente do seu grau de desenvolvimento ${ }^{1,2,6-9}$.

Essa alta incidência e prevalência na população mundial podem implicar problemas econômicos e sociais, tais como a diminuição da produtividade, altos custos do tratamento, piora da qualidade de vida e diminuição da sobrevida dos pacientes. O DM ainda contribui deforma significativa para o aparecimento de outras doenças, o que é agravado na vigência de tratamento inadequado ou ausente1, $1,2,7$.

O maior desafio para o controle da síndrome é manter a glicemia dentro de parâmetros adequados. 0 controle glicêmico almejado é glicemia dejejum menor que $126 \mathrm{mg} / \mathrm{dl}$ ehemoglobina glicosilada menor que $7 \%{ }^{10}$.

É essencial uma reorganização de hábitosalimentares para o controle do DM tipo 2. Para tanto, é necessário que haja integração entre a al imentação e os demais cuidados desenvolvidos pelo paciente. 0 comportamento alimentar é modificado deacordo com as exigências elimitações impostas pela síndrome, devendo ser revistas escolhas alimentares, diminuindo as calorias para evitar ganho de peso, aumentando a atividade física, moderando a ingestão de gordura, espaçando as refeições e monitorizando a glicemia, objetivando, finalmente, seu controle $e^{2,11,12}$.

A dieta hipocalórica, responsável pela perda ponderal, éparticularmentesignificantelogo após o diagnóstico de DM tipo 2, quando a secreção de insulina ainda é adequada. Essa dieta tem mostrado importante papel regulador no controle da hiperglicemia ainda que não haja perda ponderal, o que torna o valor da ingestão calórica mais importante que o peso em $\mathrm{Si}^{5}$.

Devido à gama de alterações do estilo de vida que são impostas às pessoas portadoras do DM tipo 2, numa fase da vida em que seus hábitos já estão bastante consolidados, a adesão destas ao tratamento tem sido um grande desafio eestefenômeno é nosso objeto de estudo neste momento.

\section{Adesão da pessoa diabética ao tratamento}

Adesão émuito mais que simplesmente cumprir determinações do profissional de saúde. Se entendida dessa maneira, supõe que o pacientenão tenha autonomia, sendo completamente excluído do controle do seu estado de saúde, cabendo esse papel exclusivamenteao profissional. Supõe também que não haja influência ou interferência de familiares, amigos ou vizinhos, nem das representações de saúde, corpo e doença para o paciente. A realidade é que, no processo de adesão, os pacientes têm autonomia ehabilidade para aceitar ou não as recomendações dos profissionais de saúde, tornando-se participantes ativos do processo de cura ${ }^{13}$.

Avaliar o processo de adesão não significa então apenas avaliar o cumprimento de determinações de profissionais de saúde, mas nos permite, embora numa perspectiva limitada, verificar fatores que tornam a incorporação de determinadas atitudes necessárias ao tratamento de saúde tão difíceis e distantes da realidade de parte dos pacientes.

Alguns fatores têm apresentado grande importância com relação à adesão dos pacientes ao tratamento. Entre eles estão respostas corporais, tais como diminuição dos episódios de hiperglicemia/hipoglicemia e diminuição dos níveis de glicemia, variáveis socioeconômicas (sexo, idade, raça, estado civil, ocupação, renda e educação), relação custo-ben efício do tratamento, interação entre médicos e pacientes, efeitos e interações medicamentosas (efeitos adversos e colaterais), concepções e conhecimentos a respeito da própria síndrome e participação da família. 0 vínculo do paciente com a unidade de saúde, contando com terapias e profissionais atualizados, também é parte importante desse process $0^{11,13,14}$.

A adesão a terapias em países desenvolvidos gira em torno de $50 \%$ e pode ser ainda muito menor em países em desenvolvimento, em face 
ao baixo acesso a serviços de saúde pela população em geral. A baixa adesão a tratamentos longos, como é o caso do diabetes, compromete a efetividade dos mesmos, tornando-se um problema crítico para a saúde da população, no tocanteà diminuição da qualidade de vida relacionada a aspectos econômicos ${ }^{15}$

Segundo Lessa ${ }^{6}$, o reconhecimento do DM tipo 2 como uma enfermidade crônica que afeta milhões de pessoas no mundo tem motivado as organizações de saúde a buscarem metodologias que favoreçam um delineamento real do problema, assim como das melhores estratégias a serem adotadas para favorecer a adesão dos pacientes ao tratamento, buscando conhecer fatores até então não considerados importantes. Segundo Aráuz $^{16}$, esses fatores se relacionam aos conhecimentos, às percepções, às atitudes, aos medos e às práticas dos pacientes em um contexto familiar e comunitário. Estes fenômenos por sua vez estão ligados às crenças das pessoas.

\section{Crenças em saúde esua relação com a adesão ao tratamento}

Deuma forma geral, no universo da saúde, podemos dizer que as crenças são definidas como idéias, conceitos, convicções eatitudes tomadas pelos pacientes que estão relacionadas à saúde ou doença e como estes fatores acabam por influir na qualidade de vida e na saúde dessas pessoas ${ }^{17}$.

Existem formas diferentes de classificação para as crenças em saúde. Rokeach ${ }^{18}$, diferencia as crenças em cinco tipos:

1) Crenças primitivas - consenso $100 \%$ - são apreendidas pelo encontro direto com o objeto da crença, reforçadas por um consenso social unânime entre todas as referências de pessoas ou grupos. Representam as verdades básicas do indivíduo sobre a realidade física, social ea natureza do eu, não sendo sujeitas à discussão ou controvérsia;

2) Crenças primitivas - consenso zero - diferem das anteriores pelo fato de que sua manutenção não depende do fato de ser compartilhada com outros: não há referência de grupos ou pessoas fora do eu que possam discutir tal crença;

3) Crenças de autoridade - são crenças inicialmente primitivas que se modificam a partir da expansão do sistema de crenças, desenvolvendo a parte não-primitiva deste. São crenças modificadas pela interação com o meio social, normalmente submetidas a uma autoridade consentida;

4) Crenças derivadas - são provenientes (ou derivadas) das crenças em determinadas autori- dades. Exemplos dessas são as crenças ideológicas, originadas mais pela identificação com a autoridade- religiosa ou política - do que com 0 objeto da crença. Ainda estão neste grupo crenças obtidas através de fontes confiáveis, tais como jornais ou revistas;

5) Crenças inconsequentes - crenças que representam questões de gosto, são mais ou menos arbitrárias. Têm pouca ligação com as outras crenças e sua modificação não traz implicação para a organização do sistema de crenças do indivíduo.

Esse modelo nos permite avaliar a força das crenças no sistema organizado pelo indivíduo, 0 que pode servir de base para a abordagem do mesmo caso deseje a modificação de determinada crença. Acredita-se que quanto mais se aproxime da crença primitiva de consenso $100 \%$, mais imutável seja, mas isso não é uma regra ${ }^{18}$.

Outra abordagem que contribui para compreender o comportamento das pessoas na área de saúde é a de Rosenstock ${ }^{19}$, cujo modelo foi proposto por Becker ${ }^{20}$. Este modelo, conhecido como modelo de crenças em saúde (MCS), inclui um forte componente de motivação e do mundo perceptual do indivíduo. É uma teoria que não só tenta explicar um problema particular de saúde, mas que também pode ser adaptada a outros contextos de comportamento ${ }^{21}$.

As características iniciais do modelo procuram prever ações e atitudes dos indivíduos em relação às doenças, tendo em vista quatro variáveis que são interdependentes ${ }^{19}$ :

1) Susceptibilidade percebida - é a variável que avalia a aceitação da possibilidade de contrair uma determinada condição ou patologia. A ênfaseé dada à realidade subjetiva e não a objetiva, explicada pelo fato de que as pessoas variam de forma acentuada na interpretação no que diz respeito a uma realidade objetiva;

2) Severidade percebida - essa variável diz respeito à percepção da seriedade de uma dada condição de saúde. 0 grau de seriedade pode ser julgado pelo grau de estimulação emocional criado pelo pensamento de uma doença, pelos tipos de dificuldade que o indivíduo acredita que uma dada condição de saúde poderá criar para ele ou mesmo pelo simples conhecimento das complicações que aquela doença poderá trazer para a sua saúde e à qualidade de vida;

3) Benefícios percebidos - o comportamento do indivíduo dependerá ainda da crença de quão benéficas, para o seu caso, serão as alternativas para reduzir a ameaça à dada condição de saúde. Deve haver pelo menos uma ação que seja subje- 
tivamente possível, seja ela relacionada à redução da susceptibilidade ou da severidade da doença;

4) Barreiras existentes - essa variável diz respeito aos aspectos negativos percebidos pelo indivíduo em relação às ações em saúde. Ele pode perceber essas ações como caras, inconvenientes, desagradáveis, dolorosas ou que exigem muito tempo disponível. Essas qualificações das ações se tornam barreiras e criam motivos conflitantes.

Para determinação dos fatores facilitadores e dificultadores da adesão, deve-se observar e estabelecer relações entre as várias instruções que são feitas ao paciente, suas ações de autocuidado, as consequências a curto, médio e longo prazo.

Entre fatores já detectados como facilitadores da adesão estão o tratamento dos pacientes por médicos especialistas, maior tempo de diagnóstico, apoio social e familiar, escolaridade do paciente, tratamento flexível, simplificação das prescrições e objetivos claros, focalizando mudanças graduais. Entre as barreiras estariam os problemas de baixa auto-estima, estresse e controle aversivo do paciente para aderir ao tratamento. A ausência de sintomas, mesmo com a síndrome já diagnosticada, pode ser também uma importante barreira ${ }^{22}$.

A piora do quadro clínico também tem uma relação com 0 aumento da adesão ao tratamento, sendo difícil verificar se existe algum patamar (medicação, exercício ou alimentação) queseja de maisfácil adesão. Existe umatendência demenor adesão à prescrição alimentar, por ser considerado o fator comportamental esocial demais difícil modificação. Quanto mais mudanças forem exigidas na rotina do paciente, mais difícil será sua adesão. Quanto menores as complicações que estiverem existindo, menor a adesão ${ }^{22}$.

Um dos fatores que tem sido pouco estudado em relação à adesão de pessoas diabéticas à terapia nutricional é a análise do seu sistema de crenças. Assim, foi desenvolvido o presente estudo, com o objetivo de analisar as crenças de pacientes portadores de diabetes tipo 2 acerca da terapia nutricional, com a finalidade de contribuir para compreender essa faceta de um fenômeno tão complexo que éa adesão.

Os achados dessa pesquisa poderão redirecionar tanto a formação dosprofissionais em saúde como sua atuação, no sentido de prestar melhor atendimento à população diabética. Além disso, podem subsidiar pesquisas futuras, no sentido de buscar a razão da formação das crenças, de modo a orientar intervenções.

\section{M etodologia}

Trata-se de um estudo de abordagem qualitativa, realizado em uma unidade de referência para atendimento de pessoas diabéticas no sistema público de saúde de uma cidade do Estado de Goiás. 0 estudo foi realizado mediante aprovação do Comitê de Ética em Pesquisa da Universidade Federal de Goiás.

0 recrutamento foi feito junto aos participantes do Programa de Diabetes da Unidade, que conta com cerca de 4.200 inscritos. N esse programa, que atende a população de toda a cidade onde se localiza e entorno, os pacientes recebem atendimento médico, nutricional e da equipe de enfermagem.

A abordagem dos participantes foi realizada uma vez por mês, às quartas-feiras, no período de novembro de 2004 a maio de 2005, no momento em que aguardavam a consulta com a nutricionista. Nesse encontro, foram prestadas informações sobre a pesquisa, suas finalidades, objetivos, procedimentos envolvidos, riscos e benefícios. Aqueles queconcordaram, assinaram termo de consentimento livree esclarecido.

A cada mês, duas ou três pessoas foram incluídas no estudo. Osúnicos critérios estabelecidos foram que a idade do participante fosse maior ou igual a dezoito anos e que o diagnóstico médico fosse diabetes tipo 2, independente de outras comorbidades existentes.

A pós o consentimento dos participantes, foi agendada data para coleta de dados que constou dos seguintes procedimentos:

- Coleta de $5 \mathrm{ml}$ de sangue venoso periférico para dosagem de hemoglobina glicosilada, em laboratório de análises clínicas conceituado, por um técnico desta instituição, respeitando as devidas medidas de biossegurança;

- Aplicação de questionário com questões para caracterização socioeconômica e cultural e entrevista registrada com auxílio de gravador, a partir de um questionário semi-estruturado, no domicílio dos participantes;

- Consulta aos prontuários na Unidade Básica de Saúde, para obtenção de informações complementares sobre o tratamento ambulatorial.

Quando prontos, os resultados de dosagem de hemoglobina glicosilada foram disponibilizados para todos os participantes, que receberam as devidas orientações sobre as taxas obtidas.

A determinação do contingente de participantes se deu a partir do momento em que ocorreu a saturação das falas, ou seja, a partir do momen- 
to em que os conteúdos das entrevistas passaram a ser repetitivos.

Para análise dos dados, após transcrição das falas, foram utilizados procedimentos de análise de conteúdo, adotando o modelo de categorias de Bardin ${ }^{23}$, modalidade temática. N esse modelo, são preconizados os seguintes passos:

1) Pré análise - fase de organização dos dados, na qual fazemos a leitura flutuante, a escoIha dos documentos, a formulação de hipóteses eobjetivos, ereferenciação dos índices e elaboração de indicadores e a preparação do material;

2) Exploração do material - consiste essencialmente em operações de codificação e enumeração do material;

3) Tratamento dos resultados, inferência e interpretação - consiste em dar aos dados significados. A partir daí, o pesquisador pode propor inferências a propósito dos objetivos ou a outras descobertas inesperadas. Em seguida, deve ser feita uma interpretação a partir de referenciais teóricos ou pragmáticos. Essa interpretação pode fornecer orientações para uma nova análise.

0 processo de categorização, para a análise de categorias, se insere neste modelo básico, sendo um processo estruturalista que comporta duas etapas:

1) Inventário - a partir de uma leitura exaustiva, isolam-se os elementos;

2) Classificação - os elementos são repartidos a partir das categorias determinadas. As categorias devem possuir as seguintes características: exclusão mútua (um elemento não pode existir em duas categorias), homogeneidade (um único princípio de classificação devenortear a organização), pertinência (adaptam-se ao material escoIhido), objetividade, fidelidade, reprodutividade.

As categorias identificadas foram interpretadas utilizando-se os referenciais de Rosenstock ${ }^{19}$ e Rokeach ${ }^{18}$.

Os resultados dos exames de hemoglobina glicosilada foram interpretados de acordo com parâmetros específicos à técnica utilizada e preconizados pelaADA ${ }^{10}$, que considera valores normais até $7,0 \%$.

\section{A presentação ediscussão dos resultados}

Participaram do estudo nove pessoas, sendo três homens e seis mulheres. Sete eram casados, um solteiro e um viúvo. Quatro eram aposentados, dois "do lar", um agente administrativo, um horticultor eum agente de saúde. A escolaridade va- riou de nenhuma a terceiro grau completo. As idades variaram entretrinta esetenta ecinco anos, a renda per capita (representada pelo total de rendimentos dos moradores da casa dividido por esse mesmo número) variou de 108 a 750 reais.

Quanto às características relacionadas à síndrome e ao tratamento em si, o tempo de diagnóstico variou de três meses a 25 anos, enquanto o tempo de atendimento na unidade de saúde variou de um mês a oito anos. 0 número de consultas médicas na unidade variou de zero a 31 , enquanto o número de consultas com nutricionista variou de um a 22.

Os resultados obtidos nos exames de hemoglobina glicosilada aplicados revelaram níveis entre 7,3 a 13,9\%, todos considerados acima do preconizado, o que, segundo $\mathrm{Cramer}^{24}$, pode significar baixa adesão ao tratamento e/ou medicação inadequada.

No processo de análise das falas, foram identificadas duas categorias, denominadas "Recomendações recebidas sobrea alimentação" e"Adesão à terapia nutricional".

Trata-se de conteúdo extremamente rico, que suscita reflexões acerca das atividades de atendimento dos profissionais de saúde, sobre a forma dos mesmos lidarem com questões de saúde em situações extremamente delicadas para os pacientes, como éo caso das doenças crônicas, queenvolvem fatores não só de "estar bem" ou "estar mal" fisicamente, mas estar adoecido, ser e existir num contexto socioeconômico, cultural efamiliar.

Recomendações recebidas

sobre a alimentação

A respeito das recomendações recebidas, pudemos observar, a partir das falas dos depoentes, que a alimentação indicada pelos profissionais ao diabético é restritiva e impositiva, carregada de expressões proibitivas como "não pode":

E comer menos coisas de açúcar e refrigerante, retirar tudo né, e massa também (P1).

Falou pra eu fazer regime, comer muita fruta, verdura (P3).

Até me deu uma tabelinha das coisas que eu não posso comer (P5).

Depois ela mandou tomar só o caldo do feijão, não pode comer o bagaço não (P6).

$M$ enos massa, comer mais verduras e legumes, e suco só à base de adoçante, e não de todas as frutas, ele recomendou mais suco de caju (P8).

As falas dos participantes são muito semeIhantes, denotando o sentido derestrição aos ali- 
mentos a serem ingeridos. Parece que o próprio profissional de saúde, ao tentar uma adesão mais significativa do paciente ao tratamento, utiliza orientações simplistas cuja tônica é a proibição.

A proposta geral para a alimentação do diabético não deveser proibitiva, esim formada por um cardápio variado, balanceado, dentro de um limite preestabelecido $0^{5,10,25}$.

Parece uma recomendação simples e fácil de ser seguida, mas dependen do dos hábitos alimentares anteriores, a limitação da quantidade de calorias pode representar uma mudança drástica e originar crenças acerca da terapia nutricional que podem ser barreira à adesão por remeter a práticas restritivas. Como já foi verificado por Torres-López ${ }^{8}$, proibir o paciente de ingerir alimentos populares e de consumo frequente, tais como o arroz e o pão, pode determinar uma baixa adesão ao tratamento. $N$ ão existe mais a indicação da redução desses alimentos para os diabéticos, abaixo do recomendado para a população no geral. A determinação da quantidade de carboidratos deve ser individualizada ${ }^{10}$.

Essas crenças podem ser classificadas como crenças de autoridade, sendo as figuras que aparecem como referência os profissi onais de saúde, que, no cenário da presente pesquisa, são das áreas de nutrição e de medicina.

As recomendações alimentares parecem advir de um processo unilateral, o paciente não é chamado para o diálogo, para a tomada de decisão em parceira, dentro desuas condições de vida.

A formação dos profissionais de saúde nesse sentido deve ser redirecionada, pois durante a fase de ensino clínico, em que os alunos exercitam o atendimento aos usuários do sistema de saúde, o que se observa ainda éa preleção, o aconselhamento, sem que o paciente seja chamado a se pronunciar sobre suas crenças, suas preferências, suas informações anteriores.

A abordagem do diabético necessita de uma visão holística, interdisciplinar, na qual haja atuação de vários profissionais de saúde, tendo em vistao controleea preven ção do diabetes, assim como a prevenção e o controle das complicações da síndrome ${ }^{2,26}$, de modo convergente e integrado.

\section{Adesão à terapia nutricional}

A necessidade de mudança de hábitos é percebida pela maioria dos participantes como parte essencial do tratamento, mas édifícil fazer essa passagem. Algumas vezes, o tipo de trabalho da pessoa pode ser uma barreira para uma alimentação mais adequada.
A verdade é a seguinte: eu achei ruim ter que comer seis vezes por dia. Por causa do serviço, eu não tenho muito tempo pra comer durante o dia (P5).

Uai, a dificuldade maior é que a gente é cozinheira, a gente fica sempre lambiscando alguma coisinha, e isso piora muito a vida da gente (P1).

Em outras vezes, atitudes da própria família é que se tornam uma barreira.

Eu tomo aquele adoçanteali, mas o meu menino, quando eu vou fazer um suco ele não gosta, então eu boto açúcar no suco e tomo junto (P3).

A participação da família, eles não ajudam e eu não esforço também porque eles fazem o que fazem, eles não têm assim aquela atenção virada para me ajudar nessa parte. Ela [a esposa] é boa pra cozinhar, ela faz, eu como. Eles comem, eu como também. $M$ as também se el es fizessem uma especial, não vou dizer quetirasse ela da rotina, masque fosse mais fácil, uma alimentação pra mim mais passiva a esse tratamento, eu acharia bom e seria mais fácil (P8).

As práticas relativas à alimentação são determinadas no convívio familiar e social. A modificação da dieta e a proposta de formação de novos hábitos al imentares devem respeitar as preferências dos indivíduos, levando em consideração seu ambientefamiliar e seu meio social. A pessoa responsável pela preparação dos alimentos para o indivíduo diabético deve estar ciente das suas necessidades e das suas preferências. Muitas vezes, é preciso ainda que outras pessoas - irmãos, pais, filhos, cônjuges - abram mão de suas práticas não saudáveis em função de uma alimentação mais adequada ao paciente diabético.

Em função de não haver acordo entre os familiares, pode ser necessária ao diabético a adoção de uma alimentação separada dos demais. Esse fator pode ser uma barreira na adesão à terapia nutricional, pois muitas vezes ele não é capaz de elaborar seus próprios alimentos, não sentemotivação para alimentar-se separadamente dos demais e acaba por não seguir as recomendações necessárias ao seu tratamento.

Por outro lado, as preferências alimentares podem ser muito poderosas:

Eu gosto de um pãozinho, não posso comer mais, queijo até que eu não acho ruim, não dou muito certo com salada e nem com fruta, eu como, mas não é aquele trem atrativo. Eu gosto de comida mais com massa, um churrasquinho, não vou mentir, carne gordurosa, eu gosto de comida bem temperadinha, bem arrumadinha, entendeu? (P5).

Os hábitos al imentares das pessoas são construídos ao longo da vida e são influenciados pelo convívio social efamiliar. A necessidade de rees- 
truturação dos hábitos alimentares dos diabéticos torna-se, por isso, uma atitude ainda mais abrangente, pois se percebe que, para que se tenha uma mudança efetiva por parte das pessoas adoecidas, é preciso que o meio no qual ela está inserida passe também por transformações.

0 que se pode observar atualmente é que os hábitos alimentares da população em geral estão inadequados. Não só em países desenvolvidos, como em países em desenvolvimento, em virtude de transformações sociais, econômicas e culturais, a forma de se alimentar e a composição da dieta das pessoas foram alteradas. Essa transformação fez com que a população passasse a ingerir mais alimentos com alto valor calórico, grandequantidade de açúcar refinado, grandequantidade de gorduras, principalmente gorduras saturadas, e maior quantidade de proteínas, enquanto que a quantidade de fibras e gorduras insaturadas diminuiu?.

Dessa forma, é necessário um processo de reeducação alimentar global, envolvendo todas as pessoas deforma geral, emboraisso seja premente para as pessoas diabéticas e seus familiares.

Às vezes a pessoa interpreta a quantidade indicada como insuficiente às suas necessidades, apresentando, assim, dificuldade de atender às exigências de reestruturação do padrão alimentar, pois sua percepção das mesmas é que são impossíveis de serem seguidas.

Eu tenho que comer pouco arroz, pouco feijão, masseeu não comer dá fraqueza, dá aquela treme deira (P3).

0 profissional deve estar atento para verificar o grau de compreensão que o paciente teve das recomendações feitas. Ele deve ser capaz de detectar possíveis distorções do quefoi recomendado. Deve existir um diálogo entre o profissional e o paciente e um espaço para que o paciente coloque suas dúvidas, caso contrário poderá ocorrer a dificuldade de adesão por interpretação errada das informações.

Alguns depoentes citam como uma dificuldade de adesão à dieta sua restrição financeira para adquirir os alimentos prescritos:

A gente não dá conta de comprar a alimentação, pra alimentar corretamente como eles mandam. Porque é muito caro as frutas. A gente come aquel as coisas que são mais baratas ( $P 4)$.

Além da dificuldade em adquirir alimentos básicos, os depoentes acrescentam a dificuldade para comprar produtos dietéticos:

Você não acha coisas para diabéticos acessíveis, você acha um açúcar, caro, você acha um sorvete, um trenzinho diferente, caro, você não tem muita opção. Pra nossa renda não tá dando certo seguir esse tipo de dieta assim (P5).

Muitas vezes, ao receber as primeiras orientações sobre a alimentação adequada, o diabético depara com sua condição financeira como uma barreira intransponível. Cabeao profissional que o acompanha estar habilitado a propor aquisições acessíveis de acordo com as condições financeiras do paciente ${ }^{8,27}$ ediscutir as possibilidades de substituiç̧̃̃es.

Algumas vezes, os entrevistados citam um processo de vaivém na adesão, demonstrando serem necessários persistência e empenho para permanecer dentro dos novos padrões:

No início é difícil, porque a gente sente muita fome, mas depois fica fácil. De vez em quando a gente comeuma coisinha fora da dieta, masémuito de vez em quando (P4).

Direi to e direto, constantemente não faço. U m pouco é comodismo meu (P8).

Fiz essa mudança de alimentação. Mas fruta eu não como não. De vez em quando a gente sai um pouquinho, mas é muito de vez em quando (P5).

As pessoas diabéticas precisam se manter em tratamento como um processo contínuo, que se for quebrado ou interrompido poderá trazer consequências indesejadas. Como essas consequências não são aparentes a princípio, os pacientes podem acreditar que pequenos excessos não serão capazes de prejudicar seu estado de saúde geral.

O profissional deve estar ciente de que, na vigência deuma condição dealteração crônica de saúde, são esperados períodos de flutuação na adesão. Deve mostrar-se sensível para compreender os limites dos outros. Entender que para o diabético o tratamento representa em certa medida o cerceamento de sua liberdade.

Para alguns, a dificuldade foi apenas inicial, sendo progressivamente superada. Para outros, a transição foi tranquila:

A dieta eu faço direto. Açúcar eu não como e nada que faz com açúcar (P6).

Não achei a dieta difícil de fazer não (P7).

Essa menor dificuldadeéverificada quando a pessoa apresenta hábitos al imentares semel hantes aos propostos no plano alimentar do diabético e, portanto, não há dificuldade para fazer adaptações menores.

Embora refiram não terem dificuldade em seguir a orientação alimentar, os entrevistados designados como P6 e P7 apresentaram valores de hemoglobina glicosilada acima do preconizado (9,1 e 13,2\%, respectivamente). Assim, pode 
ter havido incongruência entreo discurso ea prática ou incongruência de crenças, que são as percepções mais profundas e autênticas acerca do mundo real.

$\mathrm{Na}$ categoria adesão à terapia nutricional, foi identificada a crença primitiva de consenso zero de que a dieta é difícil de ser seguida, podendo constituir-se como barreira à adesão do paciente ao tratamento.

Frente à complexidade da síndrome, queenvolve alterações e complicações orgânicas, impacto pessoal, familiar e ambiental, os gestores e profissionais de saúde precisam desenvolver ações de atendimento, contando com a participação de equipes multiprofissionais que estejam voltadas para compreender a subjetividade da população, de modo a produzir estratégias para potencializar as transformações das crenças em saúde.

O termo "dieta", usado comumente tanto pe los entrevistados como pelos profissionais de saúde de modo geral, traz uma conotação de restrição. Esta palavra poderia ser substituída por plano alimentar, alimentação saudável, ou adequada, por exemplo.

Outra tônica que poderia ser adotada na relação dos profissionais com os usuários é de que a alimentação recomendada é aquela que é mais saudável, e que deveria ser adotada por todas as pessoas de modo geral, sob pena de complicações futuras para a saúde de todos.

\section{Considerações finais}

Existena área de diabetes uma grande preocupação devido às características de pandemia que a síndrome vem adquirindo. Para os profissionais de saúde, um dos maiores desafios éa baixa adesão das pessoas diabéticas ao tratamento, no qual seinserea terapia nutricional. Esteestudo decrenças dos pacientes diabéticos tipo 2 nessa área permitiu fazer algumas inferências nessa temática.

Os depoentes evidenciam crenças que denotam barreiras e não foram mencionadas crenças relativas a benefícios percebidos. Pode estar havendo um círculo vicioso, no qual a baixa adesão não favorece a obtenção de benefícios, que, por sua vez, desencoraja a adesão. Por outro lado, a terapia nutricional que relatam ter sido recomendada é percebida como proibitiva e muito restritiva, sendo muito diferente do estilo dealimentação que praticam ou praticavam usualmente.
Foram identificadas crenças primitivas, consenso $100 \%$ e consenso zero, e crenças de autoridade e derivadas. $N$ ão foram detectadas crenças inconsequentes. 0 conjunto das crenças identificadas denota baixa adesão à terapia nutricional. A predominância de crenças mais centrais pode indicar que a baixa adesão ao tratamento seja em parte definida pelo fato de que as mudanças necessárias ao indivíduo para que ele tenha sucesso no seu tratamento não estejam acontecendo por não estarem sendo abordadas de maneira adequada, no processo de atendimento em saúde.

Os resultados fornecidos por esse estudo nos remetem a um panorama que sugere que, atualmente, a terapia nutricional está de certa forma massificada, os profissionais estão presos a conceitos e valores tradicionais, que não facilitam 0 processo de conhecimento dos pacientes a respeito da terapia que está sendo utilizada. Os pacientes recebem as prescrições de forma unilateral, deixando uma lacuna onde caberia a orientação, o diálogo e a construção do conhecimento.

Eles reforçam a posição de al guns autores ao demonstrar que a falta de adesão, como refere 0 profissional de saúde, éum reflexo da abordagem inadequada que está sendo feita. Essa abordagem não considera o paciente como um todo, físico, mental, social e cultural, e não permite que seus conhecimentos prévios interajam com suas novas percepções e ensinamentos sobre a doen ça ${ }^{28}$.

N esse contexto, a educação éitem primordial no sentido de preparar o indivíduo para lidar com suas novas necessidades, garantindo sua participação efetiva no processo de prevenção da patologia e das suas complicações ${ }^{11}$.

A educação alimentar deve habilitar o paciente a tomar decisões pautadas em dados técnicos e informações científicas, pois dessa forma ele terá capacidade para traçar seus próprios objetivos no sentido de melhorar o estado geral de sua saúde e da qualidade de vida ${ }^{1,29}$.

Trata-se de um processo progressivo, que exigeflexibilidadepor parteda equipe. As mudanças vão correr de forma lenta e gradual, e pode ser que ocorram al guns retrocessos, dependendo de acontecimentos na vida do paciente, como casamento, separação, perda de entes queridos, perda de emprego, novo emprego, mudança de cidade, entre outras. Os profissionais precisam estabelecer contratos com os pacientes, como forma de promover uma relação de responsabilidades entre eles, de forma que cada avanço seja parte de um pacto de saúde estabelecido. 
0 paciente também deve se sentir livre para expressar suas dificuldades, suas insatisfações e para relatar com sinceridade os passos dados e os fatos ocorridos, sejam eles positivos ou negativos, de forma que o profissional tenha sempre uma visão transparenteeverdadeira do queacontececom ele.

0 modo de viver e pensar de cada indivíduo tem influência significativa na adesão do mesmo a qual quer tipo de tratamento. 0 seu ponto de vista e a maneira de se envolver socialmente ampliam as discussões para além da abordagem clínica.

Essa mudança prevê uma alteração na essência do sistema de saúde, quando se deve valori- zar os trabalhadores não apenas pelo volume de consultas e atendimentos realizados, mas pela qualidade do serviço prestado ao paciente ${ }^{15}$.

Conhecer a percepção do paciente reforça a perspectiva da necessidade de um atendimento mais humanizado, tal como proposto por Benevides ${ }^{30}$, em que os sujeitos (usuários), quando mobilizados, são capazes de transformar realidades, eos profissionais devem desenvolver uma atitude de valorização das dimensões subjetivas esociais, do trabalho multiprofissional, comprometendo-se em ajudar a desenvolver as capacidades de enfrentamento da população atendida na obtenção de mel hores condições de saúde.

\section{Colaboradores}

FM Pontieri trabalhou na concepção da pesquisa, elaboração do projeto, execução da coleta eanálise de dados, além da redação final e M M Bachion trabal hou na concepção da pesquisa, orientou a elaboração das atividades de pesquisa, realizou 0 refinamento do projeto, contribuiu na análise de dados e refinamento da redação final.

\section{Referências}

1. Toscano CM. As campanhas nacionais para detecção das doenças crônicas não-transmissíveis: diabetes e hipertensão arterial. Cien Saude Colet [periódico na Internet]. 2004 [acessado 2006 ago]; 9(4):[cerca de 11 p.]. Disponível em: http://www. scielo.br/scielo.php?script=sci_arttext $\&$ pid = S141381232004000400010\&Ing=pt\&nrm=iso

2. Sociedade Brasileira de Diabetes. Consensos [periódico na Internet] 2002 [acessado 2003 set 10]. Disponível em: http://www.diabetes.org.br

3. Gross JL, Vivolo SRGF, Franco L, Schmidt MI, Motta D, Quintão E, Pimazzoni Netto A. Diagnóstico e classificação do melito e tratamento do diabetes melito tipo 2. Recomendações da Sociedade Brasileira de Diabetes. Arq Bras Endocrinol M etab 2000; 44(4):S8-S27.

4. Nucci LB. A nationwide population screening program diabetes in Brazil. Revista Panamericana de Salud Pública / Pan American Journal of Public Health 2004; 16(5): 320-327.

5. Mahan LK, Escott-Stump S. Krause - alimentos, nutrição e dietoterapia. 10aed. São Paulo: Roca; 2002.

6. Lessa, I. Doenças crônicas não-transmissíveis no Brasil: um desafio para a complexa tarefa a vigilância. Cien Saude Colet [periódico na Internet] 2004 [acessado 2006 ago]; 9(4):[cerca de 13 p.]. Disponível em: http://www.scielo.br/scielo.php?script= sci_arttext\&pid=S1413-81232004000400014\&Ing= pt\&nrm=iso

7. Rocha JLL, Baggio HCC, Cunha CA, Niclewicz EA, Leite SAO, Baptista MIDK. Aspectos relevantes da interface entre diabetes mellitus e infecção. Arq Bras Endocrinol M etab 2002; 46(3):221-229.

8. Torres-López MT, Sandoval-Díaz M, Pando-M oreno M. "Sangre y azúcar": representaciones sobre la diabetes de los enfermos crónicos en un barrio de Guadalajara, M éxico. Cad Saude Publica 2005; 21(1):101-110. 
9. Sartorelli DS, Franco LJ. Tendências do diabetes mellitus no Brasil: o papel da transição nutricional. Cad Saude Publica 2003; 19(Supl 1):S29-S36.

10. American Diabetes Association. Standards of medical care in diabetes. Diabetes Care 2005; 28(Supl 1):54-536.

11. Paiva DCP, Berbusa APS, Escuder M ML. Avaliação da assistência ao paciente com diabetes e/ou hipertensão pelo Programa Saúde da Família no M unicípio de Francisco M orato, São Paulo, Brasil. Cad Saude Publica [periódico na Internet] 2006 [acessado 2006 ago]; 22(2):[cerca de 9 p.]. Disponível em: http:// www.scielo.br/scielo.php?script $=$ sci_arttext $\&$ pid $=S 0102-311 X 2006000200015 \& \mathrm{lng}=\mathrm{pt} \&$ nrm=iso

12. Davidson M B. Diabetes mellitus - diagnóstico e tratamento. 4a ed. Rio de Janeiro: Revinter; 2001.

13. Gonçalves H, Costa JSD, M enezes AM B, Knauth D, Leal OF. Adesão à terapêutica da tuberculose em Pelotas, Rio Grande do Sul: na perspectiva do paciente. Cad Saude Publica 1999; 15(4):777-787.

14. Leite SN, Vasconcellos M PC. Adesão à terapêutica medicamentosa: elementos para a discussão de conceitos e pressupostos adotados na literatura. Cien Saude Colet [periódico na Internet] 2003 [acessado 2005 ago 01]; 8(3):[cerca de 8 p.]. Disponível em: http://www.scielo.br/scielo.php?script=sci_arttext $\&$ pid $=\$ 1413-81232003000300011 \&$ Ing $=p t \& \bar{n} r m=i s o$

15. Theme-Filha M M, Szwarcwald CL, Souza-Júnior PRB. Características sócio-demográficas, cobertura de tratamento e auto-avaliação da saúde dos indivíduos que referiram seis doenças crônicas no Brasil, 2003. Cad Saude Publica [periódico na Internet]. 2005 [acessado 2006 ago]; 21:[cerca de 17 p.]. Disponível em: http://www.scielo.br/scielo.php? script=sci_arttext $\&$ pid=S0102-311X2005000700006 $\&$ Ing $=p t \& n r m=i s s o$

16. Aráuz AG, Sanchez G, Padilla G, Fernandez M, Roselló M, Guzman S. Intervención educativa comunitaria sobre la diabetes en el ámbito de la atención primaria. Revista Panamericana de Salud Pública / Pan American Journal of Public Health 2001; 9(3):145-153.

17. Pedrosa LAK. Crenças de pessoas portadoras de hanseníase sobre sua doença: base para a compreensão de suas ações em saúde [dissertação]. Ribeirão Preto (SP): Escola de Enfermagem de Ribeirão Preto; 1991.

18. Rokeach M. Crenças, virtudes e valores. Rio de Janeiro: Interciência; 1981.
19. Rosenstock IM. Historical origins of the health belief model. Health Education M onograph 1974; 2(4):328-335.

20. Becker $\mathrm{MH}$. The health belief model and personal health behavior. Health Education M onograph 1974; 2:234.

21. Lescura Y, Mamede M V. Educação em saúde: abordagem para o enfermeiro. São Paulo: Sarvier; 1990.

22. Ferreira EAP. Adesão ao tratamento em portadores de diabetes: efeitos de um treinamento em análise de contingências sobre comportamentos de autocuidado [tese]. Brasília (DF): Instituto de Psicologia da Universidade de Brasília; 2001.

23. Bardin L. Análise de conteúdo. Lisboa: Edições 70; 2000.

24. Cramer J, Pugh MJ. The influence of insulin use on glycemic control: how well do adults follow prescriptions for insulin? Diabetes Care 2005; 28(1):7883.

25. Shils ME, Goodhart R. M odern nutrition in health and disease. $9^{\text {th }}$ ed. Vol II. Philadelphia: Lippincott William and Wilkins; 2003.

26. Torres HC, Hortale VA, Schall VA. A experiência de jogos em grupos operativos na educação em saúde para diabéticos. Cad Saude Publica 2003; 19(4):10391047.

27. Cazarini RP, Zanetti $M L$, Ribeiro $K P$, Pace $A E$, Foss MC. Adesão a um grupo educativo de pessoas portadoras de diabetes mellitus: porcentagem e causas. M edicina 2002; 35(2):142-150.

28. Anderson RM, Robins LS. How do we know? Reflections on qualitative research in diabetes. Diabetes Care 1998; 21(9).

29. Andrade GKP, Silva RP, Lopes ECS. Programa de educação alimentar para pacientes diabéticos. Diabetes Clínica 2003; 7(5):351-357.

30. Benevides R, Passos E. A humanização como dimensão pública das políticas de saúde. Cien Saude Colet [periódico na Internet] 2005 [acessado 2006 set 03]; 10(3):[cerca de 11 p.]. Disponível em: http:/ /www.scielo.br/scielo.php?script=sci_arttext\&pid $=$ S1413-81232005000300014\&lng $=$ pt $\& \bar{n} r m=i s o$

Artigo apresentado em 06/11/2006

Aprovado em 14/12/2006

Versão final apresentada em 30/01/2007 